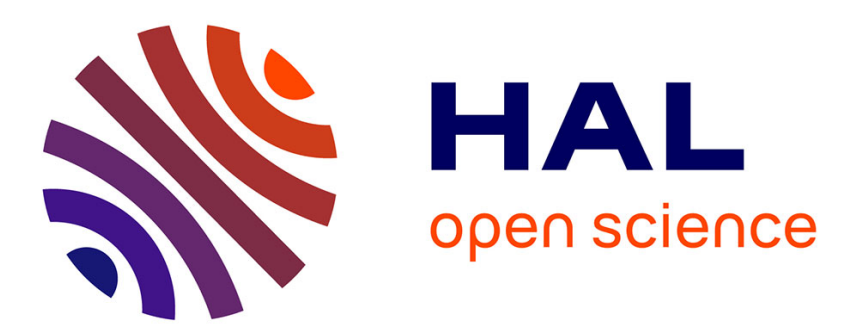

\title{
Optimal local axes and symmetry assignment for charge-density refinement
}

Slawomir Domagala, Christian Jelsch

\section{To cite this version:}

Slawomir Domagala, Christian Jelsch. Optimal local axes and symmetry assignment for charge-density refinement. Journal of Applied Crystallography, 2008, 41 (6), pp.1140-1149. 10.1107/S0021889808033384. hal-01713022

\section{HAL Id: hal-01713022 \\ https://hal.science/hal-01713022}

Submitted on 20 Feb 2018

HAL is a multi-disciplinary open access archive for the deposit and dissemination of scientific research documents, whether they are published or not. The documents may come from teaching and research institutions in France or abroad, or from public or private research centers.
L'archive ouverte pluridisciplinaire $\mathbf{H A L}$, est destinée au dépôt et à la diffusion de documents scientifiques de niveau recherche, publiés ou non, émanant des établissements d'enseignement et de recherche français ou étrangers, des laboratoires publics ou privés. 


\section{Applied \\ Crystallography}

ISSN 0021-8898

Editor: Anke R. Pyzalla

\section{Optimal local axes and symmetry assignment for charge-density refinement}

\section{Sławomir Domagała and Christian Jelsch}

J. Appl. Cryst. (2008). 41, 0

Copyright (C) International Union of Crystallography

Author(s) of this paper may load this reprint on their own web site or institutional repository provided that this cover page is retained. Republication of this article or its storage in electronic databases other than as specified above is not permitted without prior permission in writing from the IUCr.

For further information see http://journals.iucr.org/services/authorrights.html

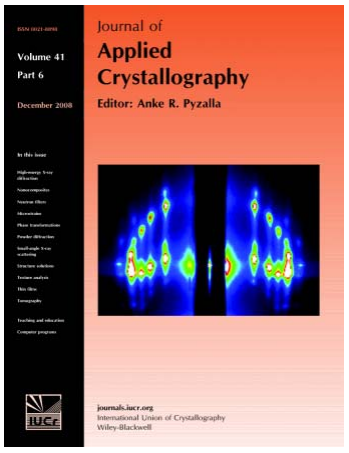

Many research topics in condensed matter research, materials science and the life sciences make use of crystallographic methods to study crystalline and non-crystalline matter with neutrons, X-rays and electrons. Articles published in the Journal of Applied Crystallography focus on these methods and their use in identifying structural and diffusioncontrolled phase transformations, structure-property relationships, structural changes of defects, interfaces and surfaces, etc. Developments of instrumentation and crystallographic apparatus, theory and interpretation, numerical analysis and other related subjects are also covered. The journal is the primary place where crystallographic computer program information is published.

\section{Crystallography Journals Online is available from journals.iucr.org}


Journal of

Applied

Crystallography

ISSN 0021-8898

Received 26 June 2008

Accepted 14 October 2008

(C) 2008 International Union of Crystallography

Printed in Singapore - all rights reserved

\section{Optimal local axes and symmetry assignment for charge-density refinement}

\author{
Sławomir Domagała and Christian Jelsch*
}

\begin{abstract}
Laboratoire de Cristallographie et Modélisation des Matériaux Minéraux et Biologiques (LCM3B) CNRS, UMR 7036, Institut Jean Barriol, Faculté des Sciences et Techniques, Nancy University, BP 239, 54506 Vandoeuvre-lès-Nancy CEDEX, France. Correspondence e-mail: christian.jelsch@uhp-nancy.fr
\end{abstract}

In the perspective of building an expanded electron-density library based on multipolar modelling for common chemical atom types, new consistent local axes systems are proposed. Optimal symmetry constraints can consequently be applied to atoms and a large number of multipole populations can be fixed to a zero value. The introduction of symmetry constraints in the multipolar refinement of small compounds allows a reduction in the number of multipolar parameters stored in the library and required for the description of the atomic electron density. In a refinement where the symmetry constraints are not applied, the use of optimal axes enables the deviations from the local pseudosymmetry to be highlighted. The application of symmetry constraints or restraints on multipoles is more effective when the axes are in accordance with the local geometry of the atom and can lead to improved crystallographic $R_{\text {free }}$ residuals. The new local axes systems, based on two or three atom neighbours, can also be usefully applied to the description of transition metal complexes.

\section{Introduction}

Advances in computers, software, and X-ray sources and detectors have made charge density more commonly available and more readily applicable to molecules of increasing size. Several libraries of multipolar atoms have been built simultaneously either from experimental data (Pichon-Pesme et al., 1995, 2004; Zarychta et al., 2007) or from theoretical calculations (Dittrich et al., 2006; Dominiak et al., 2007, and references therein). A systematic study of charge densities in a series of similar compounds was initiated by Kirschbaum et al. (2003).

Both comparative studies of charge-density parameters and database building require consistent local coordinate frames for spherical harmonic functions in a multipolar expansion. With careful choice of directions, the local coordinate system can take advantage of the spatial characteristics of certain multipoles, to model the bonding electron density derived from $s p^{2}$ and $s p^{3}$ hybrid orbitals, by the linear combination of only a few multipoles (Pichon-Pesme et al., 1995; Coppens, 1997; Jelsch et al., 2005).

Consistency in selecting the local coordinate system is also crucial for defining the atom types and subsequent averaging in a library of multipolar atoms. Proper selection of the local frame allows the imposition of symmetry restrictions on multipolar populations. This approach can considerably stabilize the initial multipole refinement, prevent the noise from being fitted and avoid an ill-conditioned normal equations matrix leading to meaningless multipole populations for non-centrosymmetric structures (El Haouzi et al., 1996; Le
Hénaff et al., 1997). A proper local axes system also enables precise analysis of the deviation of an atom from pseudosymmetry. Using the standard coordinate system, a rational comparison of various related charge-density studies can be performed. In this paper, we introduce new 'optimal' local coordinate systems for atoms in different environments. The coordinate systems are optimized in order to assign maximum symmetry to the multipoles. The symmetry constraints can be imposed or released on demand. Thus, using the optimal local axes, we describe the electron density of an atom using a minimum number of multipoles (in symmetry-constrained refinements). The few remaining multipoles that are compatible with the local symmetry generally take significant values. In symmetry-unconstrained refinements, the released multipoles generally remain small. Therefore, we can easily see which populations are most important for the proper description of the electron density. This approach is easily applied for various molecules such as small organic compounds, peptides and transition metal complexes. The optimal local axes systems were implemented and tested within our MoPro crystallographic package (Guillot et al., 2001; Jelsch et al., 2005).

\section{Methodology}

\subsection{The multipolar atom model}

Several non-spherical atom models, more elaborate than the independent atom model (IAM) approximation, have 
Table 1

Summary of the procedures used to generate the local axes systems.

\begin{tabular}{|c|c|c|c|}
\hline Axes system & Initial steps & $\begin{array}{l}\text { Orthogonalization } \\
\text { consecutive steps }\end{array}$ & Description \\
\hline$X Y$ & $\mathbf{l}_{1}=\mathbf{u}$ & $\begin{array}{l}\mathbf{l}_{3}=\mathbf{l}_{1} \times \mathbf{v} \\
\mathbf{l}_{2}=\mathbf{l}_{3} \times \mathbf{l}_{1}\end{array}$ & $\begin{array}{l}(\mathbf{u}, \mathbf{v}) \text { plane }=X Y \\
\mathbf{l}_{\mathbf{3}} \perp(\mathbf{u}, \mathbf{v}) \text { plane }\end{array}$ \\
\hline$Z X$ & $\mathbf{I}_{3}=\mathbf{u}$ & $\begin{array}{l}\mathbf{l}_{2}=\mathbf{l}_{3} \times \mathbf{v} \\
\mathbf{l}_{1}=\mathbf{l}_{2} \times \mathbf{l}_{3}\end{array}$ & $\begin{array}{l}(\mathbf{u}, \mathbf{v}) \text { plane }=Z X \\
\mathbf{l}_{2} \perp(\mathbf{u}, \mathbf{v}) \text { plane }\end{array}$ \\
\hline$b X Y$ & $\begin{array}{l}\mathbf{l}_{1}=\mathbf{u}+\mathbf{v} \\
\mathbf{l}_{2}=-\mathbf{u}+\mathbf{v}\end{array}$ & $\mathbf{l}_{3}=\mathbf{l}_{1} \times \mathbf{l}_{2}$ & $\begin{array}{l}(\mathbf{u}, \mathbf{v}) \text { plane }=X Y \\
\mathbf{l}_{\mathbf{3}} \perp(\mathbf{u}, \mathbf{v}) \text { plane }\end{array}$ \\
\hline$b Z X$ & $\begin{array}{l}\mathbf{l}_{3}=\mathbf{u}+\mathbf{v} \\
\mathbf{l}_{1}=-\mathbf{u}+\mathbf{v}\end{array}$ & $\mathbf{l}_{2}=\mathbf{l}_{3} \times \mathbf{l}_{1}$ & $\begin{array}{l}(\mathbf{u}, \mathbf{v}) \text { plane }=Z X \\
\mathbf{l}_{2} \perp(\mathbf{u}, \mathbf{v}) \text { plane }\end{array}$ \\
\hline $3 Z X$ & $\mathbf{l}_{3}=\mathbf{u}+\mathbf{v}+\mathbf{w}$ & $\begin{array}{l}\mathbf{l}_{2}=\mathbf{l}_{3} \times \mathbf{u} \\
\mathbf{l}_{1}=\mathbf{l}_{2} \times \mathbf{l}_{3}\end{array}$ & $\begin{array}{l}\mathbf{u} \in Z X \text { plane } \\
\mathbf{l}_{2} \perp Z X \text { plane }\end{array}$ \\
\hline $3 b Z$ & $\begin{array}{l}\mathbf{l}_{1}=\mathbf{v}-\mathbf{u} \\
\mathbf{l}_{2}^{\prime}=\mathbf{w}-\mathbf{u}\end{array}$ & $\begin{array}{l}\mathbf{l}_{3}=\mathbf{l}_{1} \times \mathbf{l}_{2}^{\prime} \\
\mathbf{l}_{2}=\mathbf{l}_{3} \times \mathbf{l}_{1}\end{array}$ & $\begin{array}{l}\mathbf{v}-\mathbf{u} \in Z X \text { plane } \\
\mathbf{w}-\mathbf{u} \in X Y \text { plane }\end{array}$ \\
\hline $3 Z b$ & $\begin{array}{l}\mathbf{l}_{3}=\mathbf{u} \\
\mathbf{l}_{1}^{\prime}=\mathbf{v}+\mathbf{w}\end{array}$ & $\begin{array}{l}\mathbf{l}_{2}=\mathbf{l}_{3} \times \mathbf{l}_{1}^{\prime} \\
\mathbf{l}_{1}=\mathbf{l}_{2} \times \mathbf{l}_{3}\end{array}$ & $\begin{array}{l}\mathbf{u} \in Z X \text { plane } \\
\mathbf{l}_{2} \perp Z X \text { plane }\end{array}$ \\
\hline
\end{tabular}

been proposed. Most of them are based on multipolar scattering centres which are modelled by a superposition of real spherical harmonic functions (Stewart, 1976). The Hansen \& Coppens (1978) atom model used in this study is based on this multipolar formalism and is now the most widely used in the field of small-molecule charge-density analysis:

$$
\begin{aligned}
\rho_{\text {atom }}(\mathbf{r})= & \rho_{\text {core }}(\mathbf{r})+P_{\mathrm{val}} \kappa^{3} \rho_{\mathrm{val}}(\kappa r) \\
& +\sum_{l=0}^{l_{\max }} \kappa^{\prime 3} R_{n l}\left(\kappa^{\prime} r\right) \sum_{m=0}^{l} P_{l m \pm} y_{l m \pm}(\theta, \varphi) .
\end{aligned}
$$

It describes the atomic electron density $\rho_{\text {atom }}(\mathbf{r})$ as the sum of three terms. The first two terms refer to the core and valence spherical electron density of the atom, respectively, and the third term describes the multipolar electron density as a sum of real spherical harmonic functions $y_{l m}(\theta, \varphi)$ in real form, computed with respect to a local axes system centred on the described atom.

The core and valence spherical electron densities are usually obtained from spherically averaged free-atom Hartree-Fock densities (Clementi \& Roetti, 1974). The core electron-density term is not allowed to vary (frozen core approximation), while the spherical part of the valence electron density is modulated by a valence population $P_{\text {val }}$ and the real spherical harmonic functions by multipolar populations $P_{l m}$. The $\kappa$ and $\kappa^{\prime}$ coefficients are electron-density expansioncontraction parameters. During the least-squares procedure, the $P_{\text {val }}, P_{l m}, \kappa$ and $\kappa^{\prime}$ parameters are refined against the X-ray data to fit the experimental deformation density. The $R_{n l}$ functions are Slater-type radial density functions (Slater, 1932),

$$
R_{n l}(\kappa, r)=\kappa^{3} \zeta^{n_{l}+3}(\kappa r)^{n_{l}} \exp \left(-\kappa^{\prime} \zeta r\right) /\left(n_{l}+2\right) !
$$

The sum over $l$ indices of the real spherical harmonic terms depends on the level of the multipolar expansion, which determines the number of refined multipolar parameters $P_{l m}$ needed to model adequately the deformation electron density of the chemical species. For instance, in the case of $\mathrm{C}, \mathrm{N}$ and $\mathrm{O}$ atoms, an octupolar level is applied. For heavier atom types like sulfur, phosphorous and metal elements, the expansion is set to hexadecapolar level.

The multipolar expansion for $\mathrm{H}$ atoms is generally limited to one dipole oriented along the covalent bond to model the bonding electron density directed toward the heavy atom, as usually encountered in various charge-density refinements. According to Overgaard et al. (2001), quadrupolar functions can better model the significant polarization of charge density that is observed in strong hydrogen bonds. In contrast, Mata et al. (2006) showed that the energetic properties of hydrogen bonds (total energy density at the critical point) are in better agreement with theoretical calculations when only a dipolar term is used for $\mathrm{H}$ atoms. In the present project, we aim to store multipolar parameters in a database averaged over a large number of compounds with diffraction data of unequal quality. Therefore, for the construction of the library, the multipolar expansion for $\mathrm{H}$ atoms is kept at the dipolar level.

To define the real harmonic functions needed in the multipolar formalism, local axes systems associated with each atom of the structure are generally defined. These local coordinate systems are centred on each atom of the structure and are usually oriented with respect to two of their atomic neighbours. In our previous work, including the ELMAM electron density library (Zarychta et al., 2007), the local axes used were always of type $X Y$ (Table 1). In the course of improving our experimental library and its extension from proteins/peptides to common chemical groups, new local axes systems are proposed in this study. Depending on the local geometry of the atom, the number of multipoles in the library can be significantly reduced by using optimal local axes systems with respect to the atom symmetry. All crystallographic charge-density refinements were performed with the program MoPro using all reflections $\mathbf{H}$ with an $I / \sigma(I)>0$ cutoff and a $W_{\mathbf{H}}=1 / \sigma^{2}(I)$ weighting scheme.

\subsection{Constraints and restraints}

Although it is possible to determine the experimental electron density of a compound while refining all the multipole populations, this approach can be inappropriate, depending on the quality and resolution of the diffraction data. Strong correlation between variables ( $\kappa$ and multipole population or thermal parameters) can occur, resulting in unstable refinement or unphysical values, especially for non-centrosymmetric structures (El Haouzi et al., 1996; Le Hénaff et al., 1997). To reduce the large number of parameters needed to describe the electron density for larger systems, and also to avoid the problems of high correlation between variables, several constraints for atoms can be imposed. Several types of constraints on the charge density can be applied in the MoPro program (Guillot et al., 2001; Jelsch et al., 2005).

Symmetry constraints (keyword SYMPLM in MoPro) force those multipoles that do not follow the local symmetry relation to have zero populations. For example, multipoles that are antisymmetric with respect to a symmetry mirror plane are expected to be small. Several symmetries are available, the most common being the mirrors $\left(m_{x}, m_{y}\right.$ and $\left.m_{z}\right)$, twofold and 
Table 2

Examples of chemical groups for which the local axes systems are generated.

The atom that is considered is indicated by an asterisk.

\begin{tabular}{ll}
\hline Axes system & Chemical group \\
\hline$X Y$ & $=\mathrm{O}^{*} ;=\mathrm{C}^{*} \mathrm{H}_{2} ; \mathrm{C}^{*} \mathrm{C}_{3} ; \mathrm{C}^{*}-\mathrm{CHH}$ \\
$Z X$ & $-\mathrm{C}^{*} \mathrm{H}_{3} ;-\mathrm{N}^{*} \mathrm{H}_{3}{ }^{+} ;-\mathrm{F} ;-\mathrm{H}$ \\
$b X Y$ & $\mathrm{~N}^{*} \mathrm{C}_{2} \mathrm{H}$ planar; $\mathrm{C}-\mathrm{C}^{*} \mathrm{O}_{2}{ }^{-} ; \mathrm{C}^{*}-\mathrm{CCH} ; \mathrm{O}^{*} \mathrm{H}_{2} ; \mathrm{O}^{*} \mathrm{CH}$ \\
$b Z X$ & $\mathrm{~N}^{*} \mathrm{C}_{2} \mathrm{H}$ secondary amine; $\mathrm{C}^{*} \mathrm{C}_{4} ; \mathrm{C}^{*} \mathrm{C}_{2} \mathrm{H}_{2}$ \\
$3 Z X$ & $\mathrm{~N}^{*} \mathrm{C}_{3}$ tertiary amine \\
$3 Z b$ & $M^{*} X_{4} Y$ \\
$3 b Z$ & $M^{*} X_{4}$ non-planar \\
\hline
\end{tabular}

threefold axes, and the inversion centre. Symmetry restraints are also implemented in the program. Multipoles that do not follow the symmetry can be restrained to zero with an allowed standard deviation.

Atoms of similar chemical types can be considered to have equivalent charge densities, within the library transferability approximation, by constraining their multipoles to be identical. CONPVM constraints apply to all monopoles and multipoles, CONPLM only to higher multipole populations, and CONVAL to valence monopole populations only (Jelsch et al., 2005). The differentiation of these constraints is due to their different sensitivity to atom properties. The atomic valence populations are sensitive to the chemical nature of the bonded atoms up to the second or even third connectivity shell. This is related to the different electron-withdrawing power of the chemical elements and to the electroneutrality constraint. In contrast, multipole populations depend directly

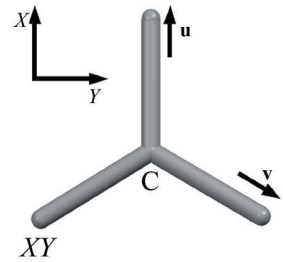

(a)

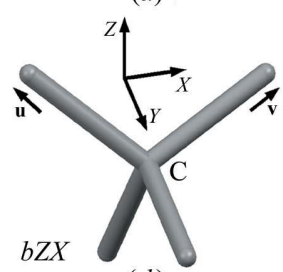

(d)

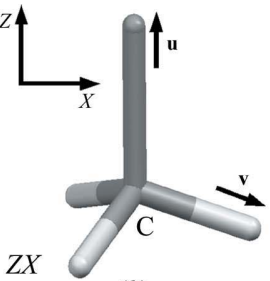

(b)

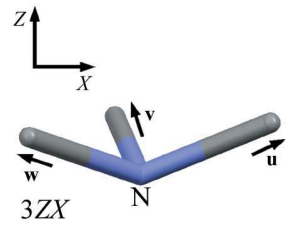

(e)

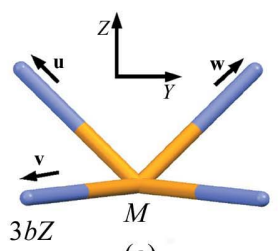

$(g)$

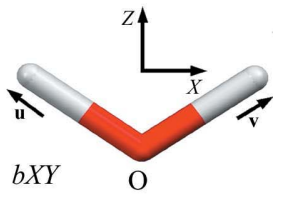

(c)

$(f)$

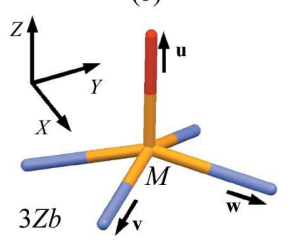

Figure 1

Representations of the seven different local axes systems defined in $\$ 2.3$. The orientations of the $\mathbf{X}, \mathbf{Y}$ and $\mathbf{Z}$ directions are shown relative to the $\mathbf{u}$, $\mathbf{v}$ and $\mathbf{w}$ vectors pointing towards neighbouring atoms. on the geometry of the atoms, i.e. bond angles, while monopoles are less sensitive. The contraction/expansion $\kappa$ and $\kappa^{\prime}$ values of two or more atoms can also be constrained to be identical via the kappa equivalence constraints CONKAP.

Analogously, it is possible to use (soft) restraints instead of (hard) constraints. The five types of constraints described above have their corresponding restraints available within MoPro. Symmetry constraints do not apply to $\mathrm{H}$ atoms, as only one dipole (and possibly a quadrupole $Q z^{2}$ ) along the $X-\mathrm{H}$ bond is considered here and an implicit cylindrical symmetry is therefore assumed. Owing to the absence of core electrons, the positions and displacement parameters of $\mathrm{H}$ atoms have a larger uncertainty. The equivalence constraints of the atoms are therefore especially well suited for $\mathrm{H}$ atoms, as their charge-density parameters are more difficult to refine.

Within the library transferability approximation, the polarization of atoms due to their immediate chemical environment is averaged over several atoms in the sample. Therefore, in the charge-density refinement aimed at building the database, equivalence and symmetry constraints are applied and maintained until the end of the refinement. Additional restraints and constraints are available within MoPro and are commonly used for $\mathrm{H}$ atoms (Jelsch et al., 2005). The $X-\mathrm{H}$ distances can be restrained/constrained to the average values obtained from neutron diffraction studies (Allen, 1986). The isotropic or anisotropic displacement parameters of the $\mathrm{H}$ atom can also be given some target values. The constraints and restraints described above can be generated and imposed automatically by MoPro for a given molecule using preparation commands.

\subsection{The different local axes systems}

The consistent use of standard properly oriented local atomic axes is encouraged for the following reasons:

(a) Axes for chemically similar atoms in different molecules and in different crystallographic environments can be similarly defined.

(b) The number of multipolar parameters can be kept minimal when imposing local chemical symmetry constraints.

(c) To build the generalized electron density library, the charge-density parameters of several atoms from different compounds are averaged. All atoms of a given chemical type need to have the same local axes definition.

(d) Atom types in a new molecule need to be identified in a standardized protocol in order to transfer the multipole parameters from the library.

For the purposes stated above, we have selected local axes systems in such a way as to describe easily the best possible maximum symmetry with the minimum number of reference atoms. We have distinguished a set of a seven different orientations of Cartesian axes $\left(\mathbf{l}_{1}, \mathbf{l}_{2}, \mathbf{l}_{3}\right)$ for local axes definition, henceforth called $X Y, Z X, b X Y, b Z X, 3 Z X, 3 Z b$ and $3 b Z$ (Figs. $1 a-1 g$, respectively). The first four systems use two neighbouring atoms, while the $3 Z X, 3 Z b$ and $3 b Z$ systems need three atoms for a proper definition of the axes. These orientations are sufficient to describe the possible symmetries 
for systems containing up to six-coordinate atoms (see Table 2).

The following procedures are used to create the local coordinate system for an atom. The $\mathbf{u}, \mathbf{v}$ and (if necessary) $\mathbf{w}$ normalized vectors represent the directions from the origin atom to its neighbours,

$$
\begin{aligned}
\mathbf{u} & =\left(\mathbf{r}_{1}-\mathbf{r}_{0}\right) /\left|\mathbf{r}_{1}-\mathbf{r}_{0}\right|, \\
\mathbf{v} & =\left(\mathbf{r}_{2}-\mathbf{r}_{0}\right) /\left|\mathbf{r}_{2}-\mathbf{r}_{0}\right|, \\
\mathbf{w} & =\left(\mathbf{r}_{3}-\mathbf{r}_{0}\right) /\left|\mathbf{r}_{3}-\mathbf{r}_{0}\right|,
\end{aligned}
$$

where $\mathbf{r}_{0}, \mathbf{r}_{1}, \mathbf{r}_{2}$ and $\mathbf{r}_{3}$ are the vectors denoting the positions of the atom under consideration and of the first, second and third neighbouring atoms, respectively. The procedures use, over several steps, the vectorial product of two vectors to generate a third vector which is perpendicular. Thus, an orthogonal basis set $\left(\mathbf{l}_{1}, \mathbf{l}_{2}, \mathbf{l}_{3}\right)$ is acquired. In the final step, the $\mathbf{l}_{i}$ vectors are normalized.

This general procedure is described in Table 1 for collinear $(X Y, Z X)$ and bisecting $(b X Y, b Z X)$ orientations and for coordinate systems using three atoms for axes definition $(3 Z X$, $3 Z b$ and $3 b Z$ ). In the case of the $X Y$ local axes system, the $\mathbf{l}_{1}$ direction is defined along the vector $\mathbf{u}$. The $\mathbf{l}_{3}$ vector is then set perpendicular to the plane defined by the vectors $\mathbf{l}_{1}$ and $\mathbf{v}$. Finally, the $\mathbf{l}_{2}$ direction is set perpendicular to the first two defined directions. The $Z X$ axes are defined analogously, but the $X Y Z$ axes are permuted. The $Z X$ axes type is used for nonplanar systems when a higher-order axis is found, as in the case of a threefold axis.

In the case of the bisecting orientation $b X Y$, the directions $\mathbf{l}_{1}$ and $\mathbf{l}_{2}$ are parallel to the sum and the difference of the $\mathbf{v}$ and u normalized vectors, respectively. These two directions are already perpendicular and correspond to the inner and exterior bisecting directions of the $(\mathbf{u}, \mathbf{v})$ bivector. The third direction, $\mathbf{l}_{3}$, is then readily defined by vectorial product. The $b Z X$ axes type is prepared similarly after permutation of $X Y Z$. The bisecting local axes systems have already been proposed by Kirschbaum et al. (2003) for some $s p^{3}$ primary, secondary, tertiary and quaternary $\mathrm{C}$ atoms in groups such as $\mathrm{CH}_{3} \mathrm{C}$, $\mathrm{CH}_{2} \mathrm{C}_{2}, \mathrm{CHC}_{3}$ and $\mathrm{CC}_{4}$. However, they used the $X Y$ axes system with the help of dummy atoms to define the proper directions.

For a description of some special symmetry in the case of three-, four-, five- or six-coordinate systems, a third atom (denoted by the $\mathbf{w}$ vector) is necessary for optimal definition of the local axes (Fig. 1). Thus, for the definition of $3 m$ and $m m 2$ point group symmetries in these systems, the following types of local axes are used: $3 Z X, 3 Z b$ and $3 b Z$.

In the $3 Z X$ orientation, the $\mathbf{l}_{3}$ vector is defined as the sum of the $\mathbf{u}, \mathbf{v}$ and $\mathbf{w}$ normalized vectors. The $\mathbf{l}_{3}$ direction defines the threefold axis of an atom with three identical neighbours (type $\left.A \_B B B\right)$. The $\mathbf{l}_{1}$ direction is set perpendicular to $\mathbf{l}_{3}$ and located in the plane defined by the vectors $\mathbf{l}_{3}$ and $\mathbf{u}$ (Table 1 ). This coordinate system can be used in the case of atoms with $C_{3 v}$ symmetry and for six-coordinate metal complexes with a threefold axis (Fig. 1e).
In the case of $3 Z b$, the $\mathbf{I}_{3}$ vector is set parallel to the $\mathbf{u}$ vector. The $\mathbf{l}_{1}^{\prime}$ intermediary vector is defined as the inner bisecting direction of the $\mathbf{v}$ and $\mathbf{w}$ vectors. $\mathbf{l}_{2}$ is then set perpendicular to $\mathbf{l}_{1}^{\prime}$ and $\mathbf{l}_{3}$. Finally, the $\mathbf{l}_{1}$ direction is made to be orthogonal via the vectorial product of $\mathbf{l}_{3}$ and $\mathbf{l}_{2}$. This orientation of axes is used to define two perpendicular planes in pentacoordinate systems (Fig. 1f).

The last orientation system, $3 b Z$, is used to define two perpendicular planes in nonplanar tetracoordinate systems (Fig. $1 g$ ). The $\mathbf{l}_{1}$ and $\mathbf{l}_{2}$ vectors are constructed as $\mathbf{v}-\mathbf{u}$ and $\mathbf{w}-\mathbf{u}$, respectively. The $\mathbf{l}_{3}$ direction is made perpendicular to the plane defined by the $\mathbf{l}_{1}$ and $\mathbf{l}_{2}$ axes. The system is then orthogonalized; $\mathbf{l}_{2}$ is obtained by the cross product of $\mathbf{l}_{3}$ and $\mathbf{l}_{1}$. Finally, in all cases, the vector triplet $\left(\mathbf{l}_{1}, \mathbf{l}_{2}, \mathbf{l}_{3}\right)$ is normalized.

\subsection{Selection criteria of atoms for local axes definitions}

At first, a connectivity list is generated for all the atoms of the molecule. For unique ordering of the neighbours of a central atom, the list of atoms considered is sorted according to the following criteria of decreasing importance:

(a) decreasing atomic numbers,

(b) increasing number of bonds,

(c) increasing atomic numbers of neighbours,

$(d)$ increasing distances to the central atom.

The type of local axes system is assigned to an atom according to the number of neighbours and the symmetry of the atom. The procedure of atom selection used for axes definition depends on each local axes system and attempts to maximize the local symmetry. In the absence of symmetry, the first atoms of the connectivity list are kept to designate the neighbouring atoms used for the local axes definition. When the atom follows a local symmetry, the atoms used for the axes definition are chosen accordingly. For example, a Csp $p^{3}$ atom of connectivity type $\mathrm{C}-\mathrm{CCON}$ is assigned a local axes system of type $b Z X$, with the two $\mathrm{C}$ atoms as neighbours. $\mathrm{H}$ atoms are, however, generally avoided as neighbours for building the local axes system, as their position is less well defined. Therefore, a $\mathrm{C}$ atom of type $\mathrm{C}-\mathrm{HHCN}$ has a $b X Y$ local axes system, but with the $\mathrm{C}$ and $\mathrm{N}$ atoms defining the axes. For $\mathrm{H}$ atoms, the $Z X$ axes system is always chosen, as it is well suited for multipole expansion to the $Q z^{2}$ quadrupole level if preferred.

The symmetry is always considered within a certain tolerance of distances, angles and planarity deviations. For example, a distorted-tetrahedral coordinated atom with four neighbours of the same chemical type can be considered as having $C_{3 v}$ symmetry with four possible orientations of threefold axes. The most promising orientation is selected, after checking the deviations for distances and angles from the ideal $C_{3 v}$ symmetry.

\subsection{Symmetry assignments}

The local symmetry of an atom is determined on the basis of its connectivity (the number and chemical type of connected atoms) and the geometric parameters describing the bonds, valence angles and planarity of the atom and its first coordination shell. The highest symmetry is always assigned using 
the conditions given above, with allowed tolerances for the deviations of the distances, angles and planarity index. Using seven different orientations of the local axes, all possible symmetry relations can be properly described for atoms with up to six connected neighbours.

For atoms with only one neighbour, the symmetry assigned depends on the chemical type. For an $\mathrm{N}$ atom with a triple bond (i.e. nitrile groups), cylindrical symmetry is introduced, whereas for sulfur and oxygen only $m m 2$ symmetry is considered, since the double bond has an elliptical shape due to the $\pi$ orbital. $\mathrm{H}$ atoms have no symmetry assigned. Since in this case only the dipole along the $Z$ axis is refined, $\mathrm{H}$ atoms in fact have cylindrical symmetry. The $Z X$ local axes system is always used for atoms with one neighbour. The first and second directions are then defined by the unique neighbour and a neighbour of the neighbour, respectively.

There are three possible symmetry relations for an atom with two bonds, depending on the value of the angle (linear or not), the bond lengths and the type of connected atoms. The lowest symmetry considered here is a mirror, since three points in space are always coplanar. The $Z X$ local axes system is used for linear groups (angle $A-\mathrm{O}-B=180^{\circ}$ ), with cylindrical and $m m 2$ as possible symmetries. For nonlinear systems, the bisecting orientation $b X Y$ is applied and the local symmetry is $m$ or $m m 2$, depending on the identity of the two neighbouring atoms.

Atoms with three bonds can be coplanar with their neighbouring atoms in the case of aromatic groups and double-bond fragments. The following point groups are possible for planar atoms: $\overline{6} m 2, m m 2$ and $m$ (Table 3 ). For nonplanar systems, the possible symmetry point groups are $3 m, m$ and 1 . For the definition of local coordinate systems in a planar fragment, the $z$ axis is always set perpendicular to the aromatic or $s p^{2}$ hybridization plane. The $X Y$ system is chosen for atoms of connectivity type $A \_B \mathrm{HH}$, while the $b X Y$ orientation is used for an atom of type $A_{-} B B C, A_{-} B B \mathrm{H}$ and $A \_B C D$ (the atom being considered is denoted by the letter $A$, letters $B, C$ and $D$ denote different neighbouring atoms, and $\mathrm{H}$ denotes hydrogen atoms.)

When the system is nonplanar, the $b Z X$ local axes are used for atoms of type $A_{-} B C D$ and $A \_B B C$, with a mirror or no symmetry. For nonplanar groups $A \_B B B$ with a threefold axis, the optimal description of the local symmetry requires the three neighbour atoms to define the $3 Z X$ local axes system (Fig. 1e). The threefold axis direction is obtained by the sum of the three unitary vectors towards the neighbouring atoms.

For $s p^{3}$ atoms with four covalent bonds, the symmetry depends on the number of identical neighbours with similar geometric features. In the case of none, two, two plus two, three and four chemically identical neighbouring atoms, the atom can be classified to the point group symmetries $1, m$, $m m 2,3 m$ and $\overline{4} 3 m$, respectively. The full set of recognized symmetries is shown in Table 3.

Local axes systems based on two atom neighbours are used whenever possible for atoms with four to six neighbours. However, there are a few exceptions (Fig. 1) where a third atom is necessary for the proper definition of two perpendi-
Table 3

The possible symmetries and local axes systems that are applicable in the program MoPro in the case of tetra-, penta- and hexacoordinate systems.

Symmetries are indicated in Hermann-Mauguin and Schoenflies notation.

\begin{tabular}{|c|c|c|c|}
\hline Tricoordinate & $\begin{array}{l}\overline{6} m 2 \\
3 m \\
m m 2 \\
m\end{array}$ & $\begin{array}{l}D_{3 h} \\
C_{3 v} \\
C_{2 v} \\
C_{s}\end{array}$ & $\begin{array}{l}X Y \\
3 Z X \\
X Y, b X Y \\
Z X, b X Y, b Z X\end{array}$ \\
\hline Tetracoordinate & $\begin{array}{l}\overline{4} 3 m \\
4 / m m m \\
\overline{4} 2 m \\
4 m m \\
3 m \\
m m m \\
m m 2 \\
2 / m \\
m \\
2\end{array}$ & $\begin{array}{l}T_{d} \\
D_{4 h} \\
D_{2 d} \\
C_{4 v} \\
C_{3 v} \\
D_{2 h} \\
C_{2 v} \\
C_{2 h} \\
C_{s} \\
C_{2}\end{array}$ & $\begin{array}{l}b Z X \\
X Y \\
b Z X \\
b Z X \\
Z X \\
X Y, b X Y \\
X Y, b X Y, b Z X, 3 b Z \\
X Y \\
X Y, b Z X \\
b Z X\end{array}$ \\
\hline Pentacoordinate & $\begin{array}{l}4 m m \\
\overline{6} m 2 \\
3 m \\
m m 2 \\
m \\
2\end{array}$ & $\begin{array}{l}C_{4 v} \\
D_{3 h} \\
C_{3 v} \\
C_{2 v} \\
C_{s} \\
C_{2}\end{array}$ & $\begin{array}{l}Z X \\
X Y \\
X Y, Z X \\
Z X, 3 Z b \\
X Y, b Z X \\
Z X\end{array}$ \\
\hline Hexacoordinate & $\begin{array}{l}m 3 m \\
4 / m m m \\
\overline{4} 2 m \\
4 m m \\
\overline{6} m 2 \\
\overline{3} m \\
3 m \\
3 \\
m m m \\
m m 2 \\
2 / m \\
m \\
2 \\
\frac{1}{1}\end{array}$ & $\begin{array}{l}O_{h} \\
D_{4 h} \\
D_{2 d} \\
C_{4 v} \\
D_{3 h} \\
D_{3 d} \\
C_{3 v} \\
C_{3} \\
D_{2 h} \\
C_{2 v} \\
C_{2 h} \\
C_{s} \\
C_{2} \\
C_{i}\end{array}$ & $\begin{array}{l}Z X \\
X Y, Z X \\
Z X \\
Z X \\
3 Z X \\
3 Z X \\
3 Z X \\
3 Z X \\
Z X, b X Y \\
Z X, b Z X \\
Z X, b Z X \\
Z X, b Z X \\
Z X, b Z X \\
b Z X\end{array}$ \\
\hline
\end{tabular}

cular planes, in the case of tetra- and pentacoordinate atoms, and in the case of threefold symmetry in trigonal prismatic configurations. The program MoPro now includes the possibility of assigning automatically the proper local axes. All commonly encountered symmetry cases are taken into consideration up to hexacoordinate atoms, including transition metal complexes (see Table 3).

In all symmetry cases, the geometry of atoms with hydrogen neighbours is treated differently, as the positions of $\mathrm{H}$ atoms and especially $\mathrm{H}-X$ distances are determined with low precision. Distances and angles involving $\mathrm{H}$ atoms are therefore not taken into account in the checking of the geometric similarity within a given tolerance. For instance, $\mathrm{C}$ atoms of $-\mathrm{CH}_{3}$ and $>\mathrm{CH}_{2}$ types are always considered to follow at least a $3 m$ or $m$ symmetry, respectively. The use of hydrogen neighbours for the definition of the local axes systems is also avoided when possible. For example, an atom with four neighbours of type $\mathrm{HH} A B$ is assigned a local axes system of type ' $b X Y A B$ ', with an $m_{z}$ mirror located between the two $\mathrm{H}$ atoms.

\subsection{Automatic assignment of symmetry constraints}

Multipole symmetry constraints can be prepared automatically in MoPro. The command 'PREP CONS SYMP 
[TOLDIS] [TOLANG] [TOLPLAN]' creates symmetry constraints (SYMPLM card) applying to the multipoles of the atoms, according to the symmetry picking rules of the spherical harmonics for the assumed point group (Kara \& KurkiSuonio, 1981). The local symmetry of an atom is determined on the basis of its connectivity (the number and chemical type of connected atoms) and the geometric parameters describing the bonds, valence angles and planarity of the atom and of its first neighbours. The planarity index $p$ for an atom with connectivity 3 is defined by a dimensionless quantity between 0 and 1 and uses the three vectors $(\mathbf{u}, \mathbf{v}, \mathbf{w})$ pointing towards the neighbours,

$$
p=|[\mathbf{u}, \mathbf{v}, \mathbf{w}]| /|\mathbf{u}||\mathbf{v}||\mathbf{w}| .
$$

The geometric parameters are allowed to vary within reasonable limits to account for small deviations from ideal symmetry; the default values for allowed deviations are $0.04 \AA$, $4^{\circ}$ and 0.1 (dimensionless and normalized to 1 ) for distances, angles and planarity, respectively. All point group symmetries are considered for atoms with connectivity numbers in the range $1-6$.

\section{Application examples}

Among the local coordinate systems described, those defined by three atoms $(3 Z X, 3 b Z, 3 Z b)$ are especially novel. No charge-density study of a compound with an atom candidate for $3 b Z$ or $3 Z b$ local axes systems was found. An extensive search of the Cambridge Structural Database [CSD, Version

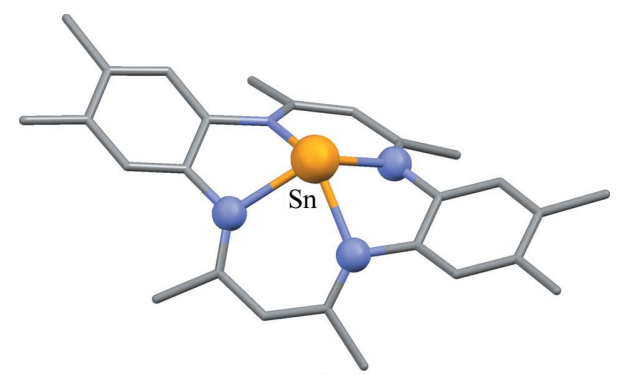

(a)

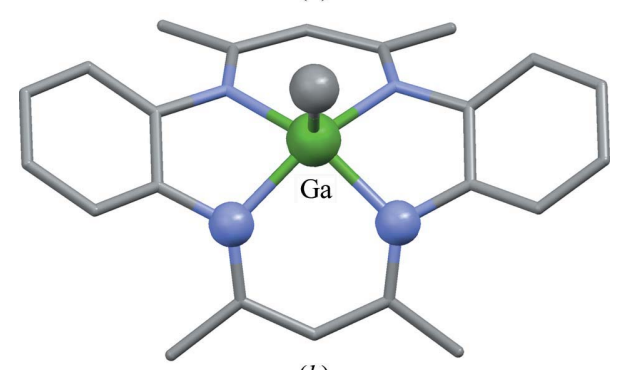

(b)

Figure 2

Two examples of transition metal complexes for the application of the new local axes based on three atoms. (a) The $3 b Z$ local axes are used for the description of the $m m 2$ symmetry of the metal atom in a tetracoordinate nonplanar complex of Sn (CSD refcode POJBIZ; Kuchta $\&$ Parkin, 1994). (b) The $3 Z b$ local axes are used for the definition of two mirror planes in a pentacoordinate nonplanar complex of Ga (CSD refcode LALTAT; Alcock et al., 1993). Atoms used for the definition of the local axes are shown as spheres. $\mathrm{H}$ atoms have been omitted for clarity.

\section{Table 4}

Multipole populations and $\kappa$ parameters for the N1 tertiary amine in morphine using different local coordinate systems.

The symmetry constraint applied to the multipoles of the $\mathrm{N} 1$ atom is indicated. When using the old $X Y$ axes system, no symmetry constraint can actually be applied.

\begin{tabular}{|c|c|c|c|c|}
\hline Refinement & I & II & III & IV \\
\hline Type of axes & $3 Z X$ & $3 Z X$ & $b X Y$ & $X Y$ \\
\hline Symmetry & 1 & $3 m$ & $m_{y}$ & 1 \\
\hline $\begin{array}{l}\text { Atoms for } \\
\text { axes }\end{array}$ & $\mathrm{C} 17, \mathrm{C} 16, \mathrm{C} 9$ & $\mathrm{C} 17, \mathrm{C} 16, \mathrm{C} 9$ & $\mathrm{C} 9, \mathrm{C} 16$ & $\mathrm{C} 17, \mathrm{C} 9$ \\
\hline$\kappa$ & $0.9653(31)$ & $0.9662(31)$ & $0.9675(31)$ & $0.9649(31)$ \\
\hline$\kappa^{\prime}$ & $0.865(28)$ & $0.870(28)$ & $0.871(28)$ & $0.864(28)$ \\
\hline$P_{\mathrm{val}}$ & $5.232(44)$ & $5.235(44)$ & $5.190(44)$ & $5.235(44)$ \\
\hline$P_{11+}$ & $-0.014(17)$ & 0 & $-0.082(16)$ & $-0.067(18)$ \\
\hline$P_{11-}$ & $-0.008(16)$ & 0 & 0 & $-0.080(16)$ \\
\hline$P_{10}$ & $-0.180(16)$ & $-0.178(16)$ & $-0.161(17)$ & $-0.148(16)$ \\
\hline$P_{20}$ & $0.106(16)$ & $0.108(16)$ & $0.090(15)$ & $0.044(14)$ \\
\hline$P_{21+}$ & 0.035 (14) & 0 & $0.063(15)$ & $0.078(14)$ \\
\hline$P_{21-}$ & $0.004(14)$ & 0 & 0 & $0.056(14)$ \\
\hline$P_{22+}$ & $-0.006(15)$ & 0 & $0.000(15)$ & $-0.004(15)$ \\
\hline$P_{22-}$ & $-0.019(15)$ & 0 & 0 & $0.020(15)$ \\
\hline$P_{30}$ & $-0.143(22)$ & $-0.146(22)$ & $0.015(18)$ & $-0.024(18)$ \\
\hline$P_{31+}$ & $0.024(17)$ & 0 & $-0.168(21)$ & $-0.097(18)$ \\
\hline$P_{31-}$ & $-0.004(18)$ & 0 & 0 & $-0.133(21)$ \\
\hline$P_{32+}$ & $0.022(19)$ & 0 & $0.059(21)$ & $-0.003(18)$ \\
\hline$P_{32-}$ & $0.021(19)$ & 0 & 0 & $0.077(21)$ \\
\hline$P_{33+}$ & $0.220(24)$ & $0.214(23)$ & $-0.175(21)$ & $0.184(23)$ \\
\hline$P_{33-}$ & $0.010(18)$ & 0 & 0 & $0.026(19)$ \\
\hline
\end{tabular}

5.29 (Allen, 2002); ConQuest, Version 1.10 (Bruno et al., 2002)] among tetraazamacrocyclic transition metal complexes resulted in four compounds satisfying the conditions for a $3 b Z$ system (CSD refcodes BAQROA, SIPYAR, VURFUJ and POJBIZ). Five compounds bearing a metal atom compatible with the $3 Z b$ system were found (refcodes HEDTIT, HUWVAW, HUWVEA, QACVUL and ALTAT). Two of these crystal structures, POJBIZ (Kuchta \& Parkin, 1994) and ALTAT (Alcock et al., 1993), are presented in Fig. 2. The allowed deviations for distances $(0.04 \AA)$, angles $\left(4^{\circ}\right)$ and planarity (0.1) were used in the search.

Several tests were conducted on selected compounds to check the validity and advantages of the constraints and local axes systems used.

\section{1. $\mathbf{N}$ atom with $3 Z X$ axes system}

The charge density of morphine hydrate, as a model compound for testing the $3 Z X$ local axes, was reanalyzed using the diffraction data from the study by Scheins et al. (2005) (Table 4). The electron-density refinements for that molecule were performed using three different local axes systems: $3 Z X$ (refinement II), $b X Y$ (refinement III) and $X Y$ (refinement IV) for the ${\mathrm{N} s p^{3}}^{3}$ atom which is bound to three $\mathrm{C}$ atoms. The three $\mathrm{N}-\mathrm{C}$ distances are very similar within $0.01 \AA$ and the three $\mathrm{C}-\mathrm{N}-\mathrm{C}$ angles within $2^{\circ}$. All these values are within the default tolerances for testing the $3 m$ symmetry constraints applied to the $\mathrm{N}$ atom. The $X Y$ system enables no local symmetry application, while the $b X Y$ local axes system allows the application of only one mirror plane to the $\mathrm{N}$ atom multipoles. The $3 Z X$ system allows the application of $3 m$ 
Table 5

Topological properties of the $\mathrm{N}-\mathrm{C}$ bonds involving the tertiary amine in morphine.

$D_{i j}$ are the bond distances, $D_{i \mathrm{CP}}$ and $D_{j \mathrm{CP}}$ are the distances of the atoms from the BCPs, $\rho\left(\mathbf{r}_{\text {bcp }}\right)$ is the total electron density and $\nabla^{2} \rho\left(\mathbf{r}_{\text {bcp }}\right)$ its Laplacian at the $\mathrm{BCP}$, and $\varepsilon$ is the ellipticity. Different local axes and symmetries were applied in the refinements. The theoretical values are taken from Scheins et al. (2005). The definitions of the refinements are explicit in Table 4.

\begin{tabular}{llllllll}
\hline Bond & Refinement & $\begin{array}{l}D_{i j} \\
(\AA)\end{array}$ & $\begin{array}{l}D_{i \mathrm{CP}} \\
(\AA)\end{array}$ & $\begin{array}{l}D_{j \mathrm{CP}} \\
(\AA)\end{array}$ & $\begin{array}{l}\rho\left(\mathbf{r}_{\text {bcp }}\right) \\
\left(\mathrm{e}^{-3}\right)\end{array}$ & $\begin{array}{l}\nabla^{2} \rho\left(\mathbf{r}_{\text {bc }}\right) \\
\left(\mathrm{e} \AA^{-5}\right)\end{array}$ & $\varepsilon$ \\
\hline $\mathrm{N} 1-\mathrm{C} 9$ & I & $1.4931(8)$ & 0.8380 & 0.6556 & 1.75 & -10.9 & 0.05 \\
& II & $1.4931(8)$ & 0.8343 & 0.6592 & 1.76 & -10.6 & 0.04 \\
& III & $1.4931(8)$ & 0.8346 & 0.6589 & 1.75 & -10.8 & 0.05 \\
& IV & $1.4931(8)$ & 0.8389 & 0.6547 & 1.75 & -10.9 & 0.05 \\
& Theory & 1.4935 & - & - & 1.72 & -15.0 & 0.04 \\
& & & & & & & \\
$\mathrm{~N} 1-\mathrm{C} 16$ & I & $1.4865(8)$ & 0.8323 & 0.6542 & 1.76 & -11.0 & 0.09 \\
& II & $1.4865(8)$ & 0.8310 & 0.6555 & 1.77 & -10.8 & 0.10 \\
& III & $1.4865(8)$ & 0.8324 & 0.6542 & 1.76 & -11.0 & 0.08 \\
& IV & $1.4865(8)$ & 0.8340 & 0.6526 & 1.76 & -11.0 & 0.09 \\
& Theory & 1.4865 & - & - & 1.74 & -15.4 & 0.07 \\
& & & & & & & \\
$\mathrm{~N} 1-\mathrm{C} 17$ & I & $1.4862(8)$ & 0.8379 & 0.6483 & 1.75 & -10.2 & 0.08 \\
& II & $1.4862(8)$ & 0.8340 & 0.6523 & 1.75 & -9.7 & 0.07 \\
& III & $1.4862(8)$ & 0.8365 & 0.6498 & 1.75 & -10.2 & 0.07 \\
& IV & $1.4862(8)$ & 0.8378 & 0.6484 & 1.75 & -10.2 & 0.08 \\
& Theory & 1.4864 & - & - & 1.73 & -15.2 & 0.05 \\
\hline
\end{tabular}

symmetry, therefore reducing the number of multipoles to be refined from 15 to four. These symmetry-constrained refinements were subsequently compared with the refinement with no symmetry applied to the multipoles of the $\mathrm{N}$ atom in the $3 Z X$ local axes system (refinement I).

The transition from one local axes system to another can be performed in principle and involves the rotation of the spherical harmonic functions (Su \& Coppens, 1994). Proper selection of the coordinate system reduces the number of refined multipoles as they become symmetry restricted. This can stabilize the refinement and prevent the noise from being fitted, or, in the case of non-centrosymmetric structures, enable the avoidance of problems due to close to singular normal equations (El Haouzi et al., 1996; Le Hénaff et al., 1997).

Refinement in the $3 Z X$ local axes system was also performed with no symmetry constraint applied to the morphine $\mathrm{N}$ atom. The values of the multipoles not respecting the pseudo- $3 m$ symmetry are generally very small, close to zero within their estimated standard deviations. These small multipole populations directly reflect the discrepancy from the symmetry in the unconstrained refinement.

The residual electron-density maps for the four refinements do not show any meaningful differences in the region of the tertiary amine $\mathrm{N}$ atom, as the highest peak and deepest hole are 0.25 and $-0.25 \mathrm{e}^{-3}$, respectively. The $R(F)$ factor is in the range $3.127-3.129 \%$ for all the refinements. The crystallographic $R(F)$ factor decreased slightly to $3.087 \%$ when all the constraints were removed after refinement II, but the highest peaks in the residual density were not changed. For comparison, the $R(F)$ factor in the original study of morphine was $2.3 \%$ with the reflections $F^{2}>2 \sigma\left(F^{2}\right)$. After applying the same cut-off, we obtained similar results for refinement II $[R(F)=2.02 \%]$. These investigations support the reason for proposing the $3 Z X$ axes system, which is compatible with pseudo-3 $m$ symmetry for that atom (Table 4 ).

The application of $3 m$ symmetry does not greatly change the values of the electron density and of its Laplacian at the
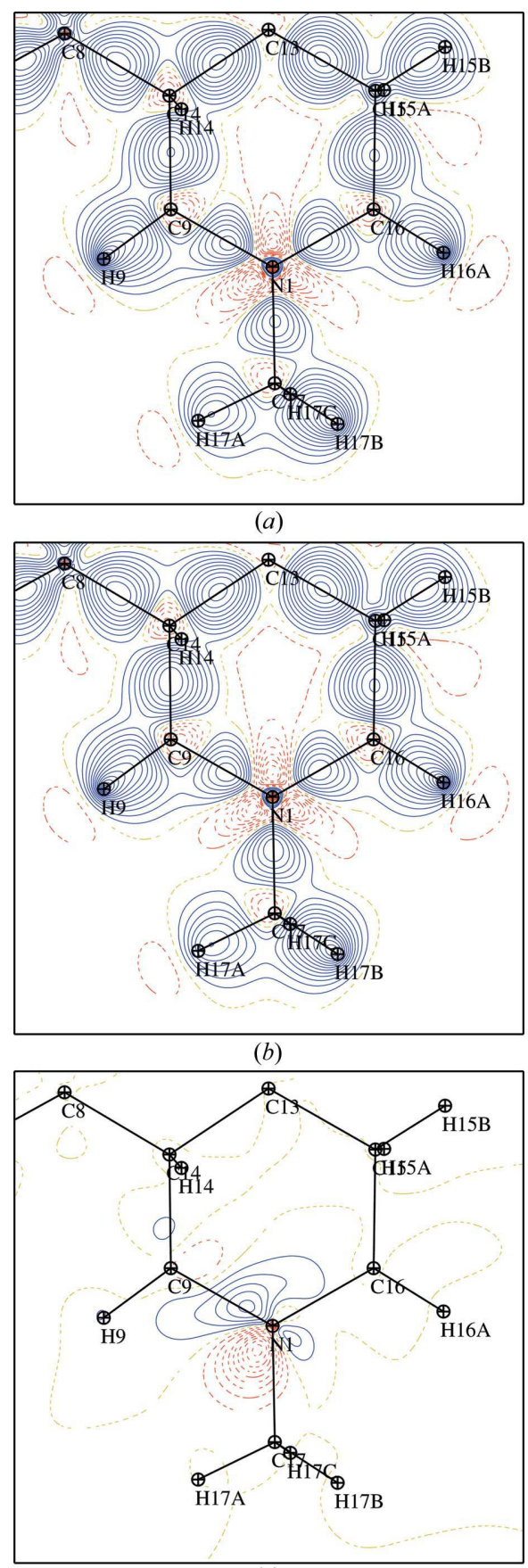

(c)

Figure 3

Deformation of the static electron density for the tertiary amine of morphine after $(a)$ unconstrained refinement I and (b) $3 m$ symmetryconstrained refinement II. The difference between refinements I and II is shown in $(c)$. A $3 Z X$ local axes system was applied to the $\mathrm{N}$ atom. The contour levels are \pm 0.05 e $\AA^{-3}$ for $(a)$ and $(b)$, and \pm 0.01 e $\AA^{-3}$ for $(c)$. Positive electron density is shown as continuous blue lines and negative as dashed red lines. The plane shown is formed by the three neighbouring $\mathrm{C}$ atoms. The level of the plane along the $\mathbf{Z}$ direction is adjusted to have the $\mathrm{N}$ atom exactly in the plane. 
Table 6

Average crystallographic statistical descriptors after refinement of the charge density of the Ala-Pro-Ala tripeptide.

The local axes systems and the application of symmetry constraints were varied. $w R^{2} F=\sum_{\mathbf{H}} W_{\mathbf{H}}\left(F_{\text {obs }}-F_{\text {calc }}\right)^{2} / \sum_{\mathbf{H}} W_{\mathbf{H}} F_{\text {obs }}^{2}$, where $W_{\mathbf{H}}=1 / \sigma^{2}\left(F_{\text {obs }}\right)^{2}$.

\begin{tabular}{lllll}
\hline Refinement & I & II & III & IV \\
\hline Local axes & Optimal & Optimal & $X Y$ & $X Y$ \\
Symmetry & Deconstrained & Constrained & Deconstrained & Constrained \\
$\left\langle w R^{2} F\right\rangle(\%)$ & $1.447(1)$ & $1.498(1)$ & $1.445(1)$ & $1.485(1)$ \\
$\left\langle w R^{2} F_{\text {free }}\right\rangle(\%)$ & $1.712(30)$ & $1.701(29)$ & $1.716(29)$ & $1.708(30)$ \\
\hline
\end{tabular}

bond critical points (BCPs) compared with the unconstrained refinement I (Table 5). The remaining nonzero multipole populations are enough to describe properly the bonding densities in the region of the $\mathrm{C}-\mathrm{N}$ bonds. The two deformation density maps resulting from the symmetry unconstrained and constrained refinements and their difference are shown in Fig. 3 in the region of the $\mathrm{N}$ atom. The difference is very small; the highest density peaks are 0.05 and $-0.09 \mathrm{e}^{-3}$ (Fig. $3 c$ ). The discrepancy from perfect $3 m$ symmetry is likely to be noise rather than chemically meaningful: the highest bonding density $\mathrm{N} 1-\mathrm{C} 9$ corresponds to the longest $\mathrm{C}-\mathrm{N}$ distance (Table 5), which is counter-intuitive.

The electron density $\rho\left(\mathbf{r}_{\text {bcp }}\right)$ and Laplacian $\nabla^{2} \rho\left(\mathbf{r}_{\text {bcp }}\right)$ values are similar to the reported experimental data and also very close to the theoretical values (Scheins et al., 2005). The relative agreement between experiment and theory was analyzed by these authors using the reliability factor $R(p)$ of a property $p$ :

$$
R(p)=\sum\left|p_{\text {exp }}-p_{\text {theo }}\right| / \sum\left|p_{\text {exp }}\right| .
$$

The values $R(\rho)=0.030$ and $R\left(\nabla^{2} \rho\right)=0.26$ were obtained from the constrained refinement II. These discrepancies are smaller than or comparable to the values of $R(\rho)=0.055$ and $R\left(\nabla^{2} \rho\right)=0.25$ in the original study of morphine.

\subsection{Ala-Pro-Ala tripeptide}

The validity and applicability of symmetry constraints was tested on the tripeptide AlaPro-Ala using the diffraction data of Kalinowski et al. (2007). The four multipole refinements were conducted with varying local axes and symmetry constraints applied to the atoms (see Table 6). The new optimal and previously used $X Y$ axes were applied, and maximum symmetry constraints were imposed or released. The other constraints (chemical equivalence) were used only for the $\mathrm{H}$ atoms for all refinements.

$5 \%$ of the reflections were selected as a test set for cross-validation of the refinement

Figure 4
(Brünger, 1992, 1993). Least-squares refinements were performed on the remaining $95 \%$ of the reflections (working set). As the free $R$ factors showed large deviations, they were averaged over several refinements with different sets of free reflections. 20 different working and test reflection sets were selected and average 'free' statistical descriptors were computed (see Table 6). The variations of the $w R^{2} F_{\text {free }}$ descriptors are very small and within their standard deviations. The $w R^{2} F$ factors decrease as expected upon release of the symmetry constraints. This is not case for the $w R^{2} F_{\text {free }}$ residuals, which are minimal for the constrained refinements. In the case of Ala-Pro-Ala, the introduction of additional refined variables (releasing symmetry constraints) does not improve the multipolar modelling of the electron density.

The behaviour of the deformation electron density for the water molecule in the two refinements can be compared in Fig. 4. The $b X Y$ local axes were assigned to the $\mathrm{O}$ atom and the $Z X$ axes to both $\mathrm{H}$ atoms. The multipole populations of

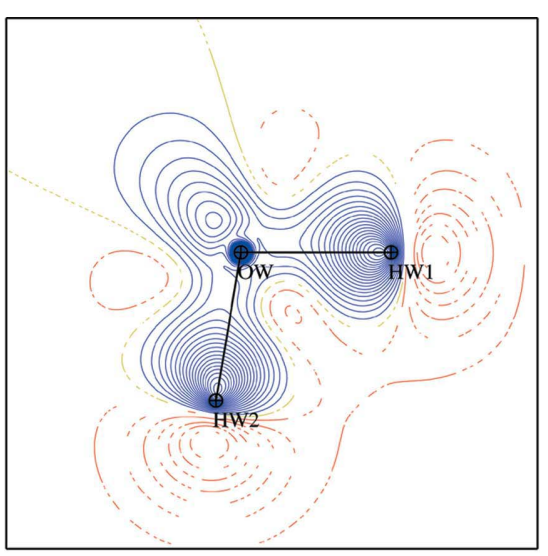

(a)

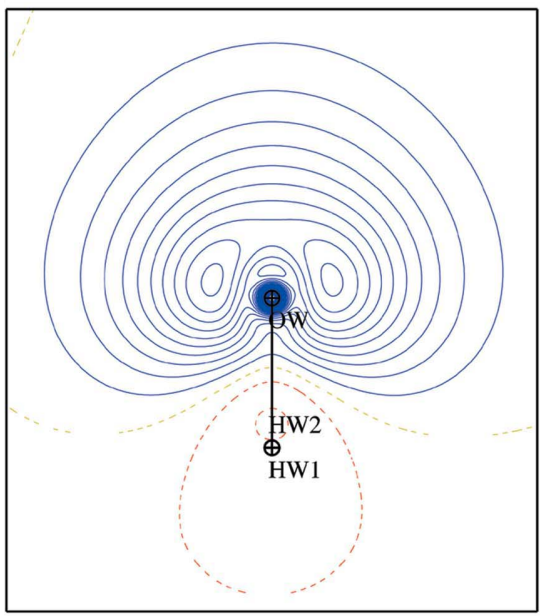

(c)

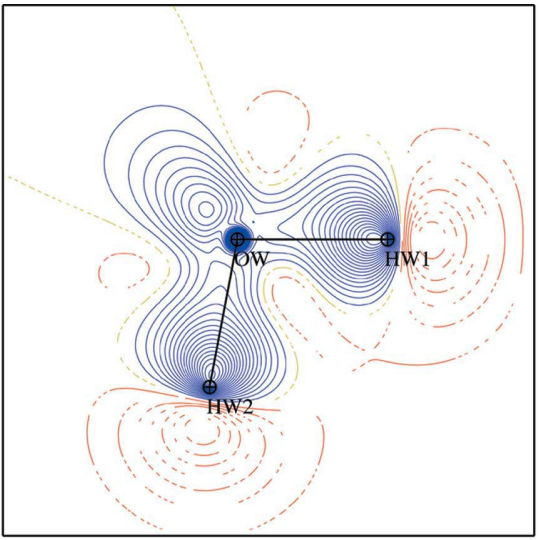

(b)

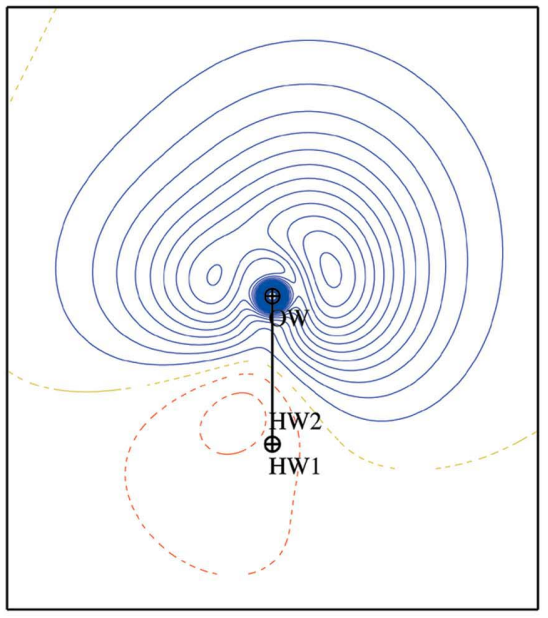

(d)

Deformation static electron-density maps for the water molecule in the tripeptide Ala-ProAla (Kalinowski et al., 2007), with different symmetry imposed on the multipoles of the $\mathrm{O}$ atom. Maps $(a)$ and $(b)$ are presented in the $\mathrm{HW} 1-\mathrm{OW}-\mathrm{HW} 2$ plane: $(a) m m 2$ symmetry, $(b)$ no symmetry. Maps $(c)$ and $(d)$ are presented in the lone pairs plane of the OW atom: $(c)$ $m m 2$ symmetry, $(d)$ no symmetry. The contour levels are $\pm 0.05 \mathrm{e}^{-3}$. Positive electron density is shown as continuous blue lines, negative as dashed red lines. 
the $\mathrm{H}$ atoms were treated as being equal. Two different levels of symmetry constraint ( $\mathrm{mm} 2$ and none) were tested on the water $\mathrm{O}$ atom. On releasing the symmetry constraints, we do not observe any visible change in the $\mathrm{H}-\mathrm{O}-\mathrm{H}$ plane (Figs. $4 a$ and $4 b$ ). However, in the perpendicular plane bisecting the $\mathrm{H}-\mathrm{O}-\mathrm{H}$ angle, there is a strong distortion of the lone pairs of the $\mathrm{O}$ atom (Figs. $4 c$ and $4 d$ ). The discrepancy of the two electron lone pairs is too strong to be realistic; the $\mathrm{O}$ atom only forms two very weak $\mathrm{N}-\mathrm{H} \cdots \mathrm{O}$ and $\mathrm{C}-\mathrm{H} \cdots \mathrm{O}$ polar interactions, the $\mathrm{H} \cdots \mathrm{O}$ distances being 2.69 and $2.57 \AA$, respectively. Therefore, the electron density of the $\mathrm{O}$ atom is better modelled using at least one mirror-plane constraint relating both electron lone pairs. Although the $X Y$ local axes system can be used for the water $\mathrm{O}$ atom, the $b X Y$ system is a better alternative as it allows two mirror planes to be applied instead of only one.

\section{3. cyclo-Pro-Ala hexapeptide}

The cyclo-Pro-Ala compound (Dittrich et al., 2002) was selected as a third example to test the new local axes systems and the symmetry constraints. Chemical equivalence constraints were applied to the $\mathrm{H}$ atoms. Additionally, the multipole populations of $\mathrm{O}=\mathrm{C}$ peptide $\mathrm{O}$ atoms were constrained together, as the deformation electron densities on the $\mathrm{O}$ atoms were unreasonable when refined independently. Four multipole refinements were conducted, with two different local axes systems ( $X Y$ and optimal) and with the symmetry constraints applied or released. In addition, we

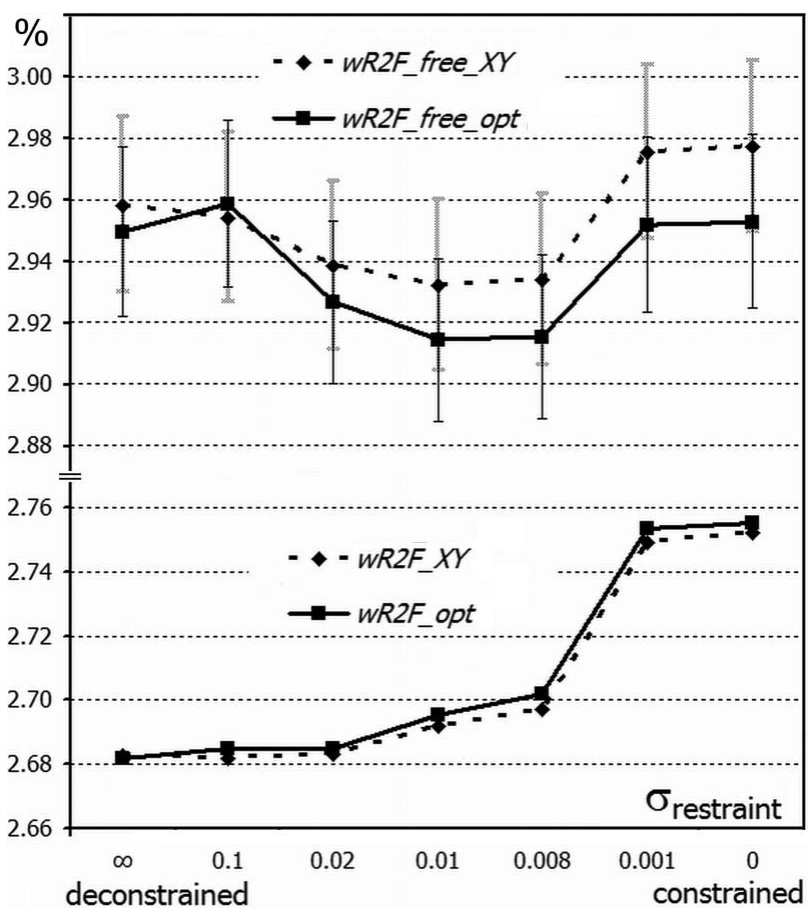

Figure 5

Crystallographic residual descriptors $w R^{2} F$ and $w R^{2} F_{\text {free }}$ for the cycloPro-Ala compound, using the optimal and $X Y$ local axes and with various multipole symmetry conditions (deconstrained, restrained, constrained). The strength of the symmetry restraints increases from left to right. performed refinements with symmetry restraints. The multipoles that do not respect the local symmetries were restrained to zero, and five different allowed standard deviations $\left(\sigma_{\text {restraint }}\right)$ were tested. Thus, a total of seven refinements were conducted for each type of axes system. We used exactly the same method of cross-validation as in the preceding example, with the same number (20) and size (5\%) of test sets for each refinement. The final residuals were averaged over 20 working and test sets.

For both local axes systems, the $w R^{2} F$ factor increases in Fig. 5, as expected, when stronger restraints are applied (lower $\left.\sigma_{\text {restraint }}\right)$. In the deconstrained refinements, this statistical factor is the same for the two axes systems. The $w R^{2} F$ factor is slightly higher for the optimal local axes for the restrained and constrained refinements, as more multipole populations are affected.

The improvement of the $R_{\text {free }}$ factors (Brünger, 1992, 1993) when applying multipolar symmetry restraints in conjunction with optimal local axes is exemplified in Fig. 5. The shape of the $w R^{2} F_{\text {free }}$ curve is typical, as it displays a minimum for moderately restrained refinements, while completely constrained or deconstrained refinements have higher free factors. In the case of deconstrained and weakly restrained refinements, almost similar free residuals are obtained with both axes systems. For the moderately restrained refinements, the $w R^{2} F_{\text {free }}$ factor decreases more strongly when using the optimal axes compared with the $X Y$ local axes. The minimum $w R^{2} F_{\text {free }}=2.915 \%$ is obtained with the optimal axes and a $\sigma_{\text {restraint }}$ in the range $0.008-0.01$ for Fig. 5. The corresponding free factor is slightly higher $(2.933 \%)$ when $X Y$ local axes are used. According to the free residual factors, it seems that joint application of the optimal local axes and of symmetry restraints improves the electron-density modelling for cycloPro-Ala in comparison with standard $X Y$ axes systems.

\subsection{Discussion}

The local axes systems described here are general and can be used in other charge-density refinement software, which generally offers the possibility of using dummy atoms for axes definition (Kirschbaum et al., 2003). When the diffraction data are of moderate quality with respect to charge-density analysis standards, the application of some symmetry constraints might be necessary, especially in the case of a non-centrosymmetric space group. The use of local axes in full accordance with the local pseudo-symmetry will render the application of multipolar symmetry constraints more powerful. Apart from library-building cases, constraints should be introduced only when necessary and should be validated by checking statistical descriptors, residual electron density, quality of deformation density maps and free $R$ factors.

For small molecular structures with high-quality diffraction data available, a constrained model leads to loss of information and is not recommended for studying certain subtle effects, e.g. intermolecular interactions. For larger molecules, especially in non-centrosymmetric cases, it is not always possible to refine all the multipolar parameters because of 
refinement reliability reasons. Thus, the optimum number of constraints to be applied depends on the centrosymmetry, and on the quality of the diffraction data and space group centrosymmetry.

On the other hand, heavily constraining a system may perturb the observation of the so-called catastrophe, as defined in the quantum theory of atoms in molecules (Bader, 1990). In topological catastrophe situations, some bond and ring critical points $(\mathrm{CP})$ coalesce, resulting in a degenerate $\mathrm{CP}$ and consequently in an unstable bond-path assignment in the structure. These catastrophe situations have already been reported for weak metal-carbon and $M \cdots \mathrm{H}$ agostic interactions (Scherer \& McGrady, 2004; Overgaard et al., 2008; Sparkes et al., 2008). Indeed, it is easy to imagine that imposing an evidently wrong symmetry or unreasonable chemical constraints may lead to a biased model.

In multipolar refinements aimed at the construction of an atomic library, a maximum number of symmetry and chemical constraints should be applied, as average charge densities are considered. As a consequence, the library contains a small number of only significant multipole populations. It is essential that standardized local axes systems are developed in order to construct generalized databases of transferable multipolar atoms. If variations in multipole parameters are to be studied for a series of compounds, it is essential that they refer to the same axes system. The previously published ELMAM database (Zarychta et al., 2007) is restricted to protein atoms and always uses the $X Y$ axes system. A new database, with the common chemical groups modelled, is under construction and is based on the local axes systems defined in this paper. One advantage of local axes aligned on the symmetry elements of the atoms will be the possibility of better analysis of deviations from the library and from the symmetry in charge-density refinements.

The ANR is gratefully acknowledged for grant No. NT053_41509 (Programme Blanc).

\section{References}

Alcock, N. W., Blacker, N. C., Errington, W. \& Wallbridge, M. G. H. (1993). Acta Cryst. C49, 1359-1361.

Allen, F. H. (1986). Acta Cryst. B42, 515-522.

Allen, F. H. (2002). Acta Cryst. B58, 380-388.
Bader, R. F. W. (1990). Atoms in Molecules: A Quantum Theory. Oxford: Clarendon Press.

Brünger, A. T. (1992). Nature (London), 355, 472-475.

Brünger, A. T. (1993). Acta Cryst. D49, 24-36.

Bruno, I. J., Cole, J. C., Edgington, P. R., Kessler, M., Macrae, C. F., McCabe, P., Pearson, J. \& Taylor, R. (2002). Acta Cryst. B58, 389397.

Clementi, E. \& Roetti, C. (1974). At. Data Nucl. Data Tables, 14, 177. Coppens, P. (1997). X-ray Charge Densities and Chemical Bonding. Oxford University Press.

Dittrich, B., Hübschle, C. B., Luger, P. \& Spackman, M. A. (2006). Acta Cryst. D62, 1325-1335.

Dittrich, B., Koritsánszky, T., Grosche, M., Scherer, W., Flaig, R., Wagner, A., Krane, H. G., Kessler, H., Riemer, C., Schreurs, A. M. M. \& Luger, P. (2002). Acta Cryst. B58, 721-727.

Dominiak, P. M., Volkov, A., Li, X. \& Coppens, P. (2007). J. Chem. Theory Comput. 3, 232-247.

El Haouzi, A., Hansen, N. K., Le Hénaff, C. \& Protas, J. (1996). Acta Cryst. A52, 291-301.

Guillot, B., Viry, L., Guillot, R., Lecomte, C. \& Jelsch, C. (2001). J. Appl. Cryst. 34, 214-223.

Hansen, N. K. \& Coppens, P. (1978). Acta Cryst. A34, 909-921.

Jelsch, C., Guillot, B., Lagoutte, A. \& Lecomte, C. (2005). J. Appl. Cryst. 38, 38-54.

Kalinowski, R., Dittrich, B., Hübschle, C. B., Paulmann, C. \& Luger, P. (2007). Acta Cryst. B63, 753-767.

Kara, M. \& Kurki-Suonio, K. (1981). Acta Cryst. A37, 201-210.

Kirschbaum, K., Poomani, K., Parrish, D. A., Pinkerton, A. A. \& Zhurova, E. (2003). J. Appl. Cryst. 36, 1464-1466.

Kuchta, M. C. \& Parkin, G. (1994). J. Am. Chem. Soc. 116, 8372-8373.

Le Hénaff, C., Hansen, N. K., Protas, J. \& Marnier, G. (1997). Acta Cryst. B53, 870-879.

Mata, I., Espinosa, E., Molins, E., Veintemillas, S., Maniukiewicz, W., Lecomte, C., Cousson, A. \& Paulus, W. (2006). Acta Cryst. A62, 365-378.

Overgaard, J., Clausen, H. F., Platts, J. A. \& Iversen, B. B. (2008). J. Am. Chem. Soc. 130, 3834-3843.

Overgaard, J., Schiøtt, B., Larsen, F. K. \& Iversen, B. B. (2001). Chem. Eur. J. 7, 3756-3767.

Pichon-Pesme, V., Jelsch, C., Guillot, B. \& Lecomte, C. (2004). Acta Cryst. A60, 204-208.

Pichon-Pesme, V., Lecomte, C. \& Lachekar, H. (1995). J. Phys. Chem. 99, 6242-6250.

Scheins, S., Messerschmidt, M. \& Luger, P. (2005). Acta Cryst. B61, 443-448.

Scherer, W. \& McGrady, G. S. (2004). Angew. Chem. Int. Ed. 43, 17821806.

Slater, J. C. (1932). Phys. Rev. 42, 33-43.

Sparkes, H. A., Brayshaw, S. K., Weller, A. S. \& Howard, J. A. K. (2008). Acta Cryst. B64, 550-557.

Stewart, R. F. (1976). Acta Cryst. A32, 565-574.

Su, Z. \& Coppens, P. (1994). Acta Cryst. A50, 636-643.

Zarychta, B., Pichon-Pesme, V., Guillot, B., Lecomte, C. \& Jelsch, C. (2007). Acta Cryst. A63, 108-125. 